\title{
Multi-component reaction on free nano-SiO 2 catalyst: Excellent reactivity combined with facile catalyst recovery and recyclability
}

\author{
MOHAMMAD ALI NASSERI* and MOHSEN SADEGHZADEH \\ Department of Chemistry, College of Sciences, Birjand University, P.O. Box 97175-615, Birjand, Iran \\ e-mail: mohammadali.nasseri@yahoo.com
}

MS received 5 May 2012; revised 6 October 2012; accepted 21 January 2013

\begin{abstract}
Nano- $\mathrm{SiO}_{2}$ catalyst was readily prepared from inexpensive starting materials in aqueous media which catalysed the synthesis $a$-aminophosphonates from aldehydes, amines and diethylphosphate under ultrasonication in water. High catalytic activity and ease of recovery from the reaction mixture using filtration, and reuse without significant losses in performance are additional eco-friendly attributes of this catalytic system.
\end{abstract}

Keywords. Nano- $\mathrm{SiO}_{2}$; ultrasonication; green catalyst; $a$-aminophosphonates; one-pot synthesis; green chemistry.

\section{Introduction}

The 'greening' of chemical processes to attain environmental friendliness ${ }^{1}$ and sustainability has become a major issue in academia and industry. Greener and environmentally sound synthetic protocols and reaction conditions have played pivotal roles in recent years toward the goal of switching to increasingly efficient and benign processes that avoid the use of volatile organic solvents, toxic reagents, hazardous and harsh reaction conditions, as well as challenging and timeconsuming wasteful separations. ${ }^{2}$ Designing organic reactions in aqueous media is another attractive area in green chemistry. ${ }^{3}$ Water is an abundant and environmentally benign solvent. As a reaction medium, it offers several benefits including control over exothermic reactions, salting in and salting out and variation of $\mathrm{pH}$. Work-up and purification can be carried out by simple phase separation techniques. Also, organic reactions in water exhibit unique reactivity and selectivity that are different from reactions in organic solvents. In particular, reactions with negative activation volume are reported to occur faster in water than in organic solvents. ${ }^{4}$ Green catalysis is a subchapter of green chemistry but probably the most important one. In most cases of organocatalysed reactions a large amount of the organocatalyst (typically, 10-30 mol\%) is needed,

*For correspondence which presents a challenge for organic chemists to utilize organocatalysts more efficiently and economically. In this context, and in view of the environmental and economical reasons, here an ongoing effort to replace such classical method with a newer method using multicomponent reactions using a nano catalyst is reported. ${ }^{5}$ In general, nano catalysts offer higher surface area and lower coordinating sites, which are responsible for the higher catalytic activity. ${ }^{6}$ Furthermore, nano catalysis has the advantage of high atom efficiency, easy product purification, and reusability of the catalyst. ${ }^{7}$ Clearly, the development of 'free' nanoparticles with tunable catalytic activity is of great significance for both academia and industry. ${ }^{8}$ Among the nanoparticle, $\mathrm{SiO}_{2}$ has been a focus of extensive research due to its chemical stability, large surface area, non-toxicity, cheap, environmentally friendly and abundant. Nano- $\mathrm{SiO}_{2}$ showed their potential in many fields, such as a catalyst or catalyst support.

$a$-Aminophosphonates have attracted much attention owing to their biological activities. Their utilities as enzyme inhibitors, antibiotics, peptide mimics, herbicides, pharmacological agents and many other applications are well-documented. ${ }^{9}$ Thus, a number of synthetic methods including enantioselective hydrophosphonylation reactions ${ }^{10}$ have been developed during the last decades. In continuation of our work on the synthesis of biologically important compounds using simple, efficient, non-toxic, and readily available catalysts, ${ }^{11-13}$ we have used of nano- $\mathrm{SiO}_{2}$ for the 


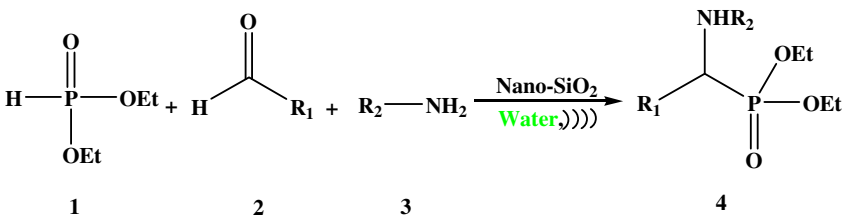

Scheme 1. Synthesis of $a$-aminophosphonates from aldehydes, amines and diethylphosphate in the presence of nano- $\mathrm{SiO}_{2}$.

synthesis of $a$-aminophosphonates from aldehydes, amines and diethylphosphate under ultrasonication in water with good to excellent yield (scheme 1).

\section{Experimental}

\subsection{General procedure}

Chemicals were purchased from Fluka and Merck in high purity. Melting points were determined in open capillaries using an Electrothermal 9100 apparatus and are uncorrected. FTIR spectra were recorded on a VERTEX 70 spectrometer (Bruker) in the transmission modein spectroscopic grade $\mathrm{KBr}$ pellets for all the powders. The particle size and structure of nano- $\mathrm{SiO}_{2}$ were observed by using a Philips CM10 transmission electron microscope operating at $100 \mathrm{KV}$. Scanning electron microscopy (SEM) studies were conducted on a Philips XL 30 scanning electron microscope. Powder $\mathrm{X}$-ray diffraction data were obtained using Bruker D8 Advance model with $\mathrm{Cu} k \alpha$ radiation. NMR spectra were recorded in $\mathrm{CDCl}_{3}$ on a Bruker Avance DRX$400 \mathrm{MHz}$ instrument spectrometer using TMS as internal standard. The purity determination of the products and reaction monitoring were accomplished by TLC on silica gel polygram SILG/UV 254 plates.

\subsection{Synthesis of nano-SiO}

The synthesis of nano- $\mathrm{SiO}_{2}$ was achieved by the ammonia-catalysed hydrolysis of tetraethyl orthosilicate (TEOS) in a mixed solvent of deionized water and ethanol using PEG as the surfactant agent in the process at room temperature. In a typical procedure, $100 \mathrm{~mL}$ ethanol and $20 \mathrm{~mL}$ deionized water were mixed together in a beaker, and then $1.0 \mathrm{~g}$ of PEG (MW 6000) was dispersed into the mixture by ultrasonication. After adding ammonia water $(2.5 \mathrm{~mL})$, tetraethyl orthosilicate (TEOS, $2 \mathrm{~mL}$ ) was added to the reaction solution. The resulting dispersion was under mechanically stirred continuously for $20 \mathrm{~h}$ at room temperature. The resultant products were collected and washed with ethanol

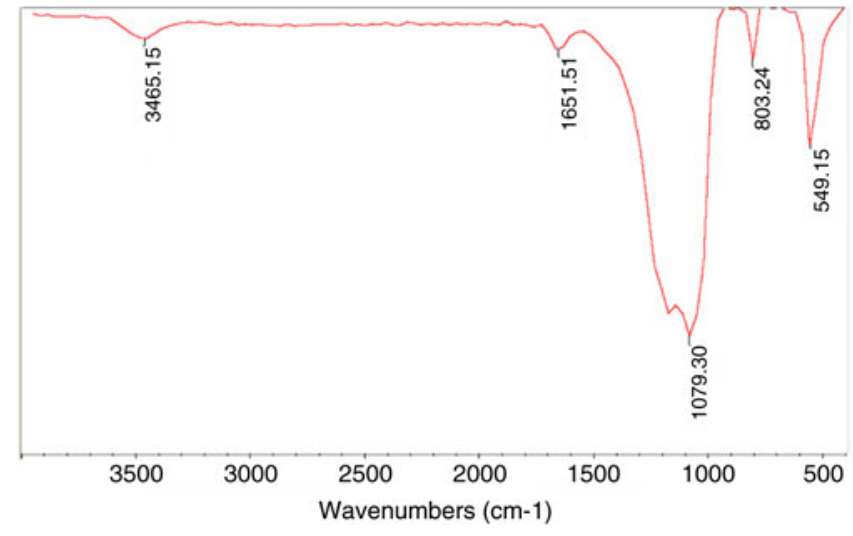

Figure 1. FT-IR spectrum of precipitated nano- $\mathrm{SiO}_{2}$.

and deionized water in sequence, and then dried under vacuum at $60^{\circ} \mathrm{C}$ for $2 \mathrm{~h}$ for further use.

\section{$2.3 \quad$ FT-IR spectroscopy}

Figure 1 shows the FT-IR spectrum of nano- $\mathrm{SiO}_{2}$. The IR band at $3465 \mathrm{~cm}^{-1}$ could be assigned to the stretching vibrations of $\mathrm{Si}-\mathrm{OH}$ groups in the structure of amorphous $\mathrm{SiO}_{2}$. Correspondingly, the IR band at $1651 \mathrm{~cm}^{-1}$ is due to the bending vibration of $\mathrm{H}_{2} \mathrm{O}$ molecules. The very strong and broad IR band at $1079 \mathrm{~cm}^{-1}$ with a shoulder at $1178 \mathrm{~cm}^{-1}$ is usually assigned to the TO and LO modes of the $\mathrm{Si}-\mathrm{O}-\mathrm{Si}$ asymmetric stretching vibrations. The IR band at $803 \mathrm{~cm}^{-1}$ can be assigned to $\mathrm{Si}-$ $\mathrm{O}-\mathrm{Si}$ symmetric stretching vibrations, whereas the IR band at $549 \mathrm{~cm}^{-1}$ is due to $\mathrm{O}-\mathrm{Si}-\mathrm{O}$ bending vibrations.

\section{$2.4 \quad X$-ray diffraction $(X R D)$ analysis}

Figure 2 shows the broadened XRD peak for amorphous silica centred at a $2 \theta=23^{\circ}$ value to our measurement. ${ }^{14}$

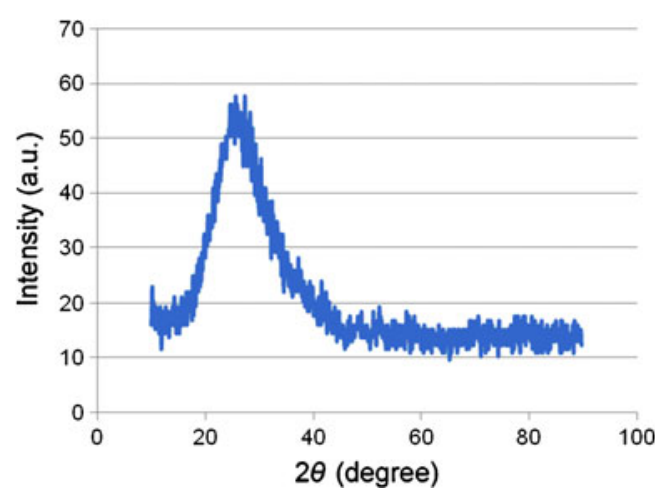

Figure 2. XRD analysis of nano- $\mathrm{SiO}_{2}$. 


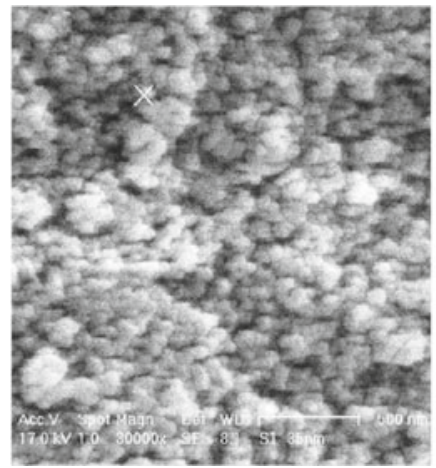

(a)

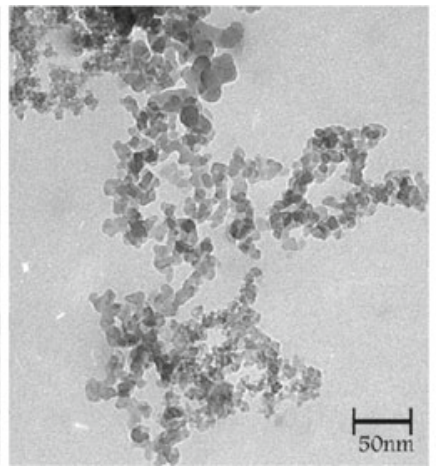

(b)

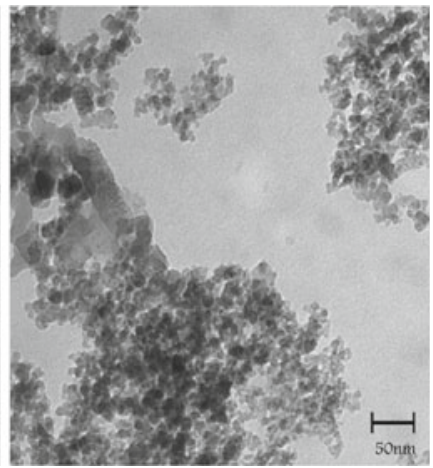

(c)

Figure 3. SEM image of nano-SiO 2 . (a) TEM images of nano-SiO 2 before use (b) and after reuse ten times. Agglomeration of silica can be seen (c).

\subsection{SEM and TEM}

The size and structure of the nano- $\mathrm{SiO}_{2}$ were also evaluated using scanning electron microscopy (SEM) and transmission electron microscopy (TEM). According to the SEM (figure 3a) and TEM (figure 3b), it was observed that the synthesized nano- $\mathrm{SiO}_{2}$ has nano dimension ranging from $\sim 20$ to $25 \mathrm{~nm}$. The results indicate that the product consists of spherical nanoparticles with smooth surfaces.

\subsection{General procedure for the synthesis of a-aminophosphonates}

A mixture of aldehydes ( $1 \mathrm{mmol})$, amines $(1 \mathrm{mmol})$, diethylphosphate $(1 \mathrm{mmol})$ and nano- $\mathrm{SiO}_{2}(0.0007 \mathrm{~g})$ in water $(5 \mathrm{ml})$, at room-temperature was irradiated by ultrasound for 10-20 min (the progress of the reaction was monitored by TLC). After completion, the catalyst was filtered and washed with $\mathrm{H}_{2} \mathrm{O}(5 \mathrm{ml})$ and $\mathrm{EtOH}(5 \mathrm{ml})$. The reaction mixture was cooled to ambient temperature and extracted with ethyl acetate $(2 \times$ $10 \mathrm{~mL}$ ). The combined organic layers were dried over $\mathrm{Na}_{2} \mathrm{SO}_{4}$, filtered and the solvent removed under reduced pressure.

\subsection{Data for compounds}

4a. ${ }^{1} \mathrm{H}$ NMR; $\delta$, ppm: $1.15(\mathrm{t}, J=6.8 \mathrm{~Hz}, 3 \mathrm{H})$, $1.32(\mathrm{t}, J=6.8 \mathrm{~Hz}, 3 \mathrm{H}), 3.64-3.72(\mathrm{~m}, 1 \mathrm{H}), 3.90$ $4.01(\mathrm{~m}, 1 \mathrm{H}), 4.04-4.19(\mathrm{~m}, 2 \mathrm{H}), 4.77(\mathrm{~d}, J=24.4 \mathrm{~Hz}$, $1 \mathrm{H}), 4.85$ (br, s, 1H), 6.59 (d, $J=8.4 \mathrm{~Hz}, 2 \mathrm{H}), 6.72$ $(\mathrm{t}, J=6.8 \mathrm{~Hz}, 1 \mathrm{H}), 7.09(\mathrm{t}, J=7.6 \mathrm{~Hz}, 2 \mathrm{H}), 7.25-$ $7.37(\mathrm{~m}, 3 \mathrm{H}), 7.48(\mathrm{~d}, J=7.6 \mathrm{~Hz}, 2 \mathrm{H}) ;{ }^{13} \mathrm{C} \mathrm{NMR} ; \delta$, ppm: 146.41, 146.23, 135.93, 129.19, 128.61, 128.59,
127.92, 127.86, 118.42, 113.89, 63.34, 63.29, 63.26, $63.22,56.82,55.35,16.45,16.39,16.19,16.14$.

4b. ${ }^{1} \mathrm{H}$ NMR; $\delta$, ppm: $1.10(\mathrm{t}, J=7.2 \mathrm{~Hz}, 3 \mathrm{H}), 1.21$ $(\mathrm{t}, J=7.2 \mathrm{~Hz}, 3 \mathrm{H}), 3.68-3.79(\mathrm{~m}, 1 \mathrm{H}), 3.89-3.97(\mathrm{~m}$, $1 \mathrm{H}), 3.99-4.11(\mathrm{~m}, 2 \mathrm{H}), 4.77(\mathrm{dd}, J=7.6 \mathrm{~Hz}, J=$ $24.4 \mathrm{~Hz}, 1 \mathrm{H}), 5.40-5.48(\mathrm{~m}, 1 \mathrm{H}), 6.41(\mathrm{~d}, J=8 \mathrm{~Hz}$, $1 \mathrm{H}), 6.55(\mathrm{t}, J=7.6 \mathrm{~Hz}, 1 \mathrm{H}), 6.69(\mathrm{t}, J=8 \mathrm{~Hz}$, $1 \mathrm{H}), 7.18-7.27(\mathrm{~m}, 4 \mathrm{H}), 7.40(\mathrm{~d}, J=7.6 \mathrm{~Hz}, 2 \mathrm{H}) ;{ }^{13} \mathrm{C}$ NMR; $\delta$, ppm: 142.39, 142.25, 135.34, 135.31, 128.59, $128.00,127.69,127.61,119.93,118.44,112.66,63.42$, $63.38,63.31,63.24,56.53,55.15,16.40,16.33,16.21$, 16.15 .

4c. ${ }^{1} \mathrm{H}$ NMR; $\delta$, ppm: $1.08(\mathrm{t}, J=7.2 \mathrm{~Hz}, 3 \mathrm{H})$, $1.27(\mathrm{t}, J=7.2 \mathrm{~Hz}, 3 \mathrm{H}), 3.60-3.68(\mathrm{~m}, 1 \mathrm{H}), 3.88$ $3.96(\mathrm{~m}, 1 \mathrm{H}), 4.05-4.21(\mathrm{~m}, 2 \mathrm{H}), 4.75(\mathrm{dd}, J=8.0 \mathrm{~Hz}$, $J=24.4 \mathrm{~Hz}, 1 \mathrm{H}), 5.37(\mathrm{br}, \mathrm{s}, 1 \mathrm{H}), 6.45(\mathrm{dd}, J=$ $2.0 \mathrm{~Hz}, J=8.4 \mathrm{~Hz}, 1 \mathrm{H}), 6.60-6.64(\mathrm{~m}, 2 \mathrm{H}), 6.97(\mathrm{t}$, $J=8 \mathrm{~Hz}, 1 \mathrm{H}), 7.26-7.34(\mathrm{~m}, 3 \mathrm{H}), 7.48(\mathrm{~d}, J=7.2 \mathrm{~Hz}$, $2 \mathrm{H}) ;{ }^{13} \mathrm{C}$ NMR; $\delta$, ppm: 147.88, 147.72, 135.59, 134.82, $130.13,128.69,128.58,128.11,128.06,127.92$, $127.86,118.09,113.80,111.82,63.82,63.40,63.29$, $63.23,56.58,55.06,16.49,16.43,16.22,16.18$.

4d. ${ }^{1} \mathrm{H}$ NMR; $\delta$, ppm: $1.12(\mathrm{t}, J=6.8 \mathrm{~Hz}, 3 \mathrm{H}), 1.28$ $(\mathrm{t}, J=6.8 \mathrm{~Hz}, 3 \mathrm{H}), 3.63-3.74(\mathrm{~m}, 1 \mathrm{H}), 3.87-3.96(\mathrm{~m}$, $1 \mathrm{H}), 4.09-4.22(\mathrm{~m}, 2 \mathrm{H}), 4.73(\mathrm{~d}, J=24 \mathrm{~Hz}, 1 \mathrm{H}), 5.22$ (br, s, 1H), $6.57(\mathrm{~d}, J=8.8 \mathrm{~Hz}, 1 \mathrm{H}), 7.04(\mathrm{~d}, J=$ $9.2 \mathrm{~Hz}, 2 \mathrm{H}), 7.27-7.34(\mathrm{~m}, 3 \mathrm{H}), 7.49(\mathrm{~d}, J=7.6 \mathrm{~Hz}$, $2 \mathrm{H}) ;{ }^{13} \mathrm{C}$ NMR; $\delta$, ppm: $147.88,147.70,135.55,134.82$, $130.13,128.65,128.57,128.11,128.06,127.92$, $127.86,118.09,113.83,111.81,63.49,63.40,63.30$, $63.23,56.56,55.08,16.51,16.45,16.23,16.18$. 
4e. ${ }^{1} \mathrm{H}$ NMR; $\delta$, ppm: $1.09(\mathrm{t}, J=6.8 \mathrm{~Hz}, 3 \mathrm{H}), 1.27$ $(\mathrm{t}, J=6.8 \mathrm{~Hz}, 3 \mathrm{H}), 3.62-3.72(\mathrm{~m}, 1 \mathrm{H}), 3.87-3.97(\mathrm{~m}$, $1 \mathrm{H}), 4.05-4.19(\mathrm{~m}, 2 \mathrm{H}), 4.71(\mathrm{~d}, J=24.4 \mathrm{~Hz}, 1 \mathrm{H})$, 4.99 (br, s, 1H), 6.53-6.56 (m, 2H), 6.78 (t, $J=8.8 \mathrm{~Hz}$, 2H), 7.22-7.33 (m, 3H), $7.48(\mathrm{~d}, J=7.2 \mathrm{~Hz}, 2 \mathrm{H}) ;{ }^{13} \mathrm{C}$ NMR; $\delta$, ppm: 146.01, 144.87, 135.43, 129.00, 128.10, $128.07,127.85,127.82,127.79,123.02,115.00,63.48$, $63.41,63.36,63.29,56.90,55.40,16.46,16.40,16.21$, 16.15 .

4f. ${ }^{1} \mathrm{H}$ NMR; $\delta$, ppm: $1.13(\mathrm{t}, J=7.2 \mathrm{~Hz}, 3 \mathrm{H})$, $1.34(\mathrm{t}, J=7.2 \mathrm{~Hz}, 3 \mathrm{H}), 3.59-3.71(\mathrm{~m}, 1 \mathrm{H}), 3.87-$ $3.98(\mathrm{~m}, 1 \mathrm{H}), 4.12-4.23(\mathrm{~m}, 2 \mathrm{H}), 4.43(\mathrm{dd}, J=$ $7.6 \mathrm{~Hz}, J=24 \mathrm{~Hz}, 1 \mathrm{H}), 6.35(\mathrm{br} s, 1 \mathrm{H}), 6.44(\mathrm{~d}$, $J=9.2 \mathrm{~Hz}, 1 \mathrm{H}), 7.28-7.36(\mathrm{~m}, 3 \mathrm{H}), 7.51(\mathrm{~d}, J=$ $7.2 \mathrm{~Hz}, 2 \mathrm{H}), 8.00(\mathrm{~d}, J=9.2 \mathrm{~Hz}, 2 \mathrm{H}) ;{ }^{13} \mathrm{C} \mathrm{NMR} ; \delta$, ppm: $152.25,138.83,134.44,128.89,128.58,128.52$, $128.49,127.86,127.81,126.05,112.42,63.92,63.85$, $63.40,63.33,56.24,54.73,16.48,16.40,16.22,16.14$.

4g. ${ }^{1} \mathrm{H}$ NMR; $\delta$, ppm: $1.15(\mathrm{t}, J=7.2 \mathrm{~Hz}, 3 \mathrm{H}), 1.35$ $(\mathrm{t}, J=7.2 \mathrm{~Hz}, 3 \mathrm{H}), 2.23(\mathrm{~s}, 3 \mathrm{H}), 3.68-3.76(\mathrm{~m}, 1 \mathrm{H})$, 3.94-4.03 (m, 1H), 4.09-4.21 (m, 2H), $4.79(\mathrm{~d}, J=$ $24 \mathrm{~Hz}, 1 \mathrm{H}), 6.59$ (d, $J=8 \mathrm{~Hz}, 2 \mathrm{H}), 6.96(\mathrm{~d}, J=8 \mathrm{~Hz}$, $2 \mathrm{H}), 7.29-7.32(\mathrm{~m}, 1 \mathrm{H}), 7.39(\mathrm{t}, J=7.4 \mathrm{~Hz}, 2 \mathrm{H}), 7.52$ $(\mathrm{d}, J=7.2 \mathrm{~Hz}, 2 \mathrm{H}) ;{ }^{13} \mathrm{C} \mathrm{NMR} ; \delta$, ppm: $136.06,129.66$, $128.67,128.57,127.88,127.82,127.60,113.98,63.32$, $63.27,63.24,63.19,57.13,55.63,20.39,16.49,16.43$, $16.24,16.18$.

4h. ${ }^{1} \mathrm{H}$ NMR; $\delta$, ppm: $1.15(\mathrm{t}, J=6.8 \mathrm{~Hz}, 3 \mathrm{H})$, $1.30(\mathrm{t}, J=6.8 \mathrm{~Hz}, 3 \mathrm{H}), 3.67(\mathrm{~s}, 3 \mathrm{H}), 3.69-3.78(\mathrm{~m}$, $1 \mathrm{H}), 3.87-3.99(\mathrm{~m}, 1 \mathrm{H}), 4.09-4.21(\mathrm{~m}, 2 \mathrm{H}), 4.74(\mathrm{~d}$, $J=24 \mathrm{~Hz}, 1 \mathrm{H}), 6.59(\mathrm{~d}, J=8.8 \mathrm{~Hz}, 2 \mathrm{H}), 6.70(\mathrm{~d}$, $J=8.8 \mathrm{~Hz}, 2 \mathrm{H}), 7.24-7.29(\mathrm{~m}, 1 \mathrm{H}), 7.34(\mathrm{t}, J=$ $7.4 \mathrm{~Hz}, 2 \mathrm{H}), 7.45(\mathrm{~d}, J=7.2 \mathrm{~Hz}, 2 \mathrm{H}) ;{ }^{13} \mathrm{C} \mathrm{NMR} ; \delta$, ppm: 152.66, 140.46, 140.32, 136.08, 128.58, 127.93, $127.88,115.24,114.70,63.35,63.28,63.24,63.18$, $57.71,56.22,55.60,29.68,16.44,16.38,16.20,16.14$.

4i. ${ }^{1} \mathrm{H}$ NMR; $\delta$, ppm: $1.17(\mathrm{t}, J=7.2 \mathrm{~Hz}, 3 \mathrm{H})$, $1.32(\mathrm{t}, J=7.2 \mathrm{~Hz}, 3 \mathrm{H}), 3.60-3.75(\mathrm{~m}, 1 \mathrm{H}), 3.78(\mathrm{~s}$, $3 \mathrm{H}), 3.91-3.99(\mathrm{~m}, 1 \mathrm{H}), 4.05-4.23(\mathrm{~m}, 2 \mathrm{H}) 4.82(\mathrm{dd}$, $J=7.6 \mathrm{~Hz}, J=23.2 \mathrm{~Hz}, 1 \mathrm{H}), 6.38(\mathrm{br}, \mathrm{s}, 1 \mathrm{H})$, $6.63(\mathrm{~d}, J=9.2 \mathrm{~Hz}, 2 \mathrm{H}), 6.87(\mathrm{~d}, J=8.4 \mathrm{~Hz}, 2 \mathrm{H})$, $7.42(\mathrm{~d}, 2 \mathrm{H}), 7.98(\mathrm{~d}, J=9.2 \mathrm{~Hz}, 2 \mathrm{H}) ;{ }^{13} \mathrm{C} \mathrm{NMR} ; \delta$, ppm: $159.68,152.65,152.31,152.19,138.72,129.05$, $129.00,126.42$, 126.00, 114.29, 112.38, 63.85, 63.78, $63.31,63.25,55.15,54.56,22.63,16.44,16.40,16.28$, $16.22,14.10$. 4j. ${ }^{1} \mathrm{H}$ NMR; $\delta$, ppm: $1.12(\mathrm{t}, J=7.09 \mathrm{~Hz}, 3 \mathrm{H})$, 1.35 (t, $J=7.09 \mathrm{~Hz}, 3 \mathrm{H}), 3.76-3.89(\mathrm{~m}, 1 \mathrm{H}), 3.93-$ $4.03(\mathrm{~m}, 1 \mathrm{H}), 4.14-4.27(\mathrm{~m}, 2 \mathrm{H}), 5.05(\mathrm{br}, \mathrm{s}, 1 \mathrm{H})$, $6.21(\mathrm{dd}, J=3.8 \mathrm{~Hz}, J=22.8 \mathrm{~Hz}, 1 \mathrm{H}), 6.70(\mathrm{~d}$, $J=8 \mathrm{~Hz}, 2 \mathrm{H}), 6.77(\mathrm{t}, J=7.2 \mathrm{~Hz}, 1 \mathrm{H}), 7.17-$ $7.20(\mathrm{~m}, 2 \mathrm{H}), 7.43-7.47(\mathrm{~m}, 1 \mathrm{H}), 7.58(\mathrm{t}, J=8 \mathrm{~Hz}$, $1 \mathrm{H}), 7.78-7.80(\mathrm{~m}, 1 \mathrm{H}), 8.01(\mathrm{~d}, J=8 \mathrm{~Hz}, 2 \mathrm{H}) ;{ }^{13} \mathrm{C}$ NMR; $\delta$, ppm: 149.49, 149.42, 145.48, 145.31, 133.52, $133.50,131.96,129.42,128.92,128.77,128.56$, $128.53,125.28,118.86,113.61,63.94,63.87,63.42$, $63.35,50.68,49.20,16.41,16.35,15.99,15.91$.

4k. ${ }^{1} \mathrm{H}$ NMR; $\delta$, ppm: $1.23(\mathrm{t}, J=6.8 \mathrm{~Hz}, 3 \mathrm{H}), 1.34$ (t, $J=6.8 \mathrm{~Hz}, 3 \mathrm{H}), 3.86-3.97(\mathrm{~m}, 1 \mathrm{H}), 4.03-4.11(\mathrm{~m}$, $1 \mathrm{H}), 4.13-4.26(\mathrm{~m}, 2 \mathrm{H}), 4.86(\mathrm{~d}, J=25.2 \mathrm{~Hz}, 1 \mathrm{H})$, $6.55(\mathrm{~d}, J=7.6 \mathrm{~Hz}, 2 \mathrm{H}), 6.70-6.79(\mathrm{~m}, 1 \mathrm{H}), 7.10-7.15$ $(\mathrm{m}, 2 \mathrm{H}), 7.69-7.72(\mathrm{~m}, 2 \mathrm{H}), 8.20-8.24(\mathrm{~m}, 2 \mathrm{H}) ;{ }^{13} \mathrm{C}$ NMR; $\delta$, ppm: 147.64, 146.82, 146.68, 144.15, 129.38, $128.72,123.76,119.10,113.84,63.80,63.70,63.47$, $63.40,56.42,54.50,16.45,16.41,16.27,16.21$.

4l. ${ }^{1} \mathrm{H}$ NMR; $\delta$, ppm: $1.22(\mathrm{t}, J=7.2 \mathrm{~Hz}, 3 \mathrm{H}), 1.34$ $(\mathrm{t}, J=7.2 \mathrm{~Hz}, 3 \mathrm{H}), 3.86-3.95(\mathrm{~m}, 1 \mathrm{H}), 4.01-4.10$ $(\mathrm{m}, 1 \mathrm{H}), 4.11-4.27(\mathrm{~m}, 2 \mathrm{H}), 4.81(\mathrm{~d}, J=24.8 \mathrm{~Hz}$, $1 \mathrm{H}), 5.15$ (br, s, 1H), $6.64(\mathrm{~d}, J=8 \mathrm{~Hz}, 2 \mathrm{H}), 6.70$ $(\mathrm{t}, J=7.6 \mathrm{~Hz}, 1 \mathrm{H}), 7.10(\mathrm{t}, J=7.8 \mathrm{~Hz}, 2 \mathrm{H}), 7.30$ $(\mathrm{d}, J=8 \mathrm{~Hz}, 2 \mathrm{H}), 7.43-7.46(\mathrm{~m}, 2 \mathrm{H}) ;{ }^{13} \mathrm{C} \mathrm{NMR} ; \delta$, ppm: $146.19,146.05,134.65,133.71,129.25,129.20$, $128.82,128.78,118.63,113.88,63.51,63.45,63.40$, $63.31,56.25,54.92,16.46,16.41,16.31,16.24$.

4m. ${ }^{1} \mathrm{H}$ NMR; $\delta$, ppm: $1.18(\mathrm{t}, J=6.8 \mathrm{~Hz}, 3 \mathrm{H})$, $1.32(\mathrm{t}, J=6.8 \mathrm{~Hz}, 3 \mathrm{H}), 2.33(\mathrm{~s}, 3 \mathrm{H}), 3.68-3.79$ $(\mathrm{m}, 1 \mathrm{H}), 3.93-4.04(\mathrm{~m}, 1 \mathrm{H}), 4.10-4.20(\mathrm{~m}, 2 \mathrm{H}), 4.75-$ $4.86(\mathrm{~m}, 2 \mathrm{H}), 6.64(\mathrm{~d}, J=7.7 \mathrm{~Hz}, 2 \mathrm{H}), 6.74(\mathrm{t}$, $J=7.2 \mathrm{~Hz}, 1 \mathrm{H}), 7.11-7.19(\mathrm{~m}, 4 \mathrm{H}), 7.38-7.41(\mathrm{~m}$, $2 \mathrm{H}) ;{ }^{13} \mathrm{C}$ NMR; $\delta$, ppm: $146.51,146.37,137.63,137.58$, $132.78,129.38,129.34,129.32,129.24,129.17$, $127.79,127.71,118.32,113.85,63.32,63.24,63.20$, $63.17,56.54,55.00,21.18,16.50,16.42,16.26,16.20$.

4n. ${ }^{1} \mathrm{H}$ NMR; $\delta$, ppm: $1.15(\mathrm{t}, J=7.2 \mathrm{~Hz}, 3 \mathrm{H}), 1.26$ $(\mathrm{t}, J=7.2 \mathrm{~Hz}, \mathrm{H}), 3.76(\mathrm{~s}, 3 \mathrm{H}), 3.90-3.99(\mathrm{~m}, 1 \mathrm{H})$, $4.06-4.22(\mathrm{~m}, 2 \mathrm{H}), 4.78(\mathrm{dd}, J=5.6 \mathrm{~Hz}, J=24 \mathrm{~Hz}$, 1H), 4.96 (br, s, 1H), 6.65 (d, $J=7.6 \mathrm{~Hz}, 2 \mathrm{H}), 6.69$ $(\mathrm{t}, J=7.2 \mathrm{~Hz}, 1 \mathrm{H}), 6.88(\mathrm{~d}, J=8.4 \mathrm{~Hz}, 2 \mathrm{H}), 7.11$ $(\mathrm{t}, J=8 \mathrm{~Hz}, 2 \mathrm{H}), 7.40-7.43(\mathrm{~m}, 2 \mathrm{H}) ;{ }^{13} \mathrm{C} \mathrm{NMR} ; \delta$, ppm: 159.32, 159.27, 146.53, 146.39, 129.10, 129.03, 128.95, 118.24, 114.01, 113.87, 63.25, 63.15, 56.05, $55.12,46.53,16.45,16.38,16.28,16.22$. 


\section{Results and discussion}

The reaction was carried out in various solvents under similar conditions. In this study, it was found that water is a more efficient (table 1, entry 1 ) over other organic solvents (table 1, entries 2-13) with respect to reaction time and yield of the desired $a$-aminophosphonates. The reaction proceeded perfectly in polar solvents (table 1, entries 1, 2, 3, 7, 10, 11), but the yields decreased when the reaction was carried out in nonpolar solvents (table 1, entries 8, 9, 12).

At this stage, We have studied the catalyst concentration on model reaction. The catalyst plays a crucial role in the success of the reaction in terms of the rate and the yields. For example, diethylphosphate reacts with benzaldehyde and benzenamine in the presence of $0.0003 \mathrm{~g}$ nano- $\mathrm{SiO}_{2}$ in water giving the product of $78 \%$ yield. Increasing the percentage of catalyst to $0.0005 \mathrm{~g}$ and $0.0007 \mathrm{~g}$ resulted in the yields $91 \%$ and $97 \%$, respectively. Higher amounts of the catalyst did not improve the results to a greater extent. Thus, $0.0007 \mathrm{~g}$ nano- $\mathrm{SiO}_{2}$ was chosen as the maximum quantity of catalyst used for these reactions (figure 4).

The activity of the recycled catalyst was also examined under the optimized conditions. After the completion of reaction, nano- $\mathrm{SiO}_{2}$ was removed by filtration, washed with ethanol and dried at the pump. The recovered catalyst was reused for ten consecutive cycles without any significant loss in catalytic activity,

Table 1. Solvent screening for the reaction between benzaldehyde, benzenamine and diethylphosphate. ${ }^{\mathrm{a}}$

\begin{tabular}{llc}
\hline Entry & Solvent & ${\text { Yield }(\%)^{\mathrm{b}}}^{\mathrm{b}}$ \\
\hline 1 & $\mathrm{H}_{2} \mathrm{O}$ & 97 \\
2 & $\mathrm{EtOH}_{3}$ & 69 \\
3 & $\mathrm{CH}_{3} \mathrm{CN}$ & 64 \\
4 & $\mathrm{THF}$ & 52 \\
5 & $\mathrm{CH}_{2} \mathrm{Cl}_{2}$ & 46 \\
6 & Toluene & 20 \\
7 & EtOAC & 75 \\
8 & $n$-Hexane & Trace \\
9 & $\mathrm{CHCl}$ & Trace \\
10 & DMSO & 70 \\
11 & MeOH & 72 \\
12 & Dioxane & Trace \\
13 & DMF & 64 \\
14 & Solvent-free & 76 \\
\hline
\end{tabular}

${ }^{a}$ Reaction conditions: benzaldehyde $(1 \mathrm{mmol})$, benzenamine ( $1 \mathrm{mmol})$, diethylphosphate $(1 \mathrm{mmol})$, solvent $(10 \mathrm{ml})$, nanosilica $(0.001 \mathrm{~g})$ at room-temperature under ultrasonication for $30 \mathrm{~min}$

${ }^{\mathrm{b}}$ Isolated yields

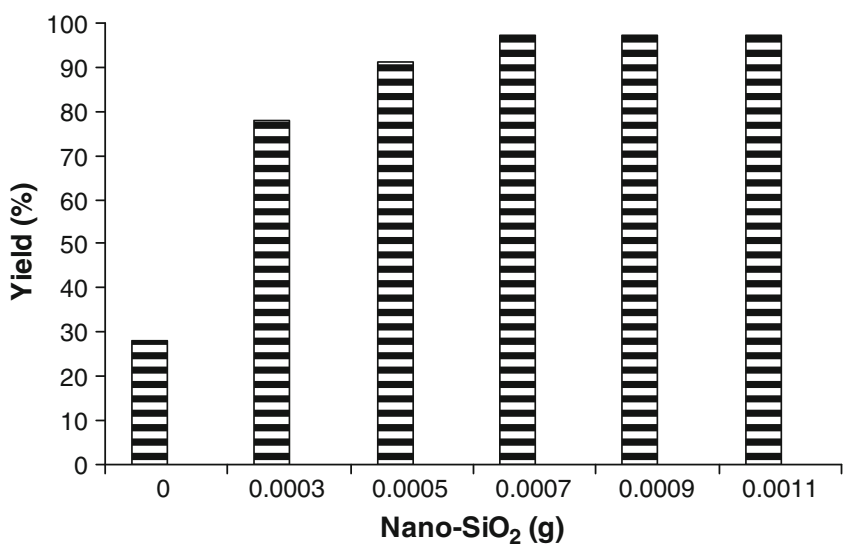

Figure 4. Effect of increasing amount of nano- $\mathrm{SiO}_{2}$ on the preparation of $a$-aminophosphonates. Reaction of diethylphosphate, benzaldehyde and benzenamine in the presence of nano- $\mathrm{SiO}_{2}$ at room temperature under ultrasonication in water.

proving its robustness (TEM; figure 5). The characterization of the nano- $\mathrm{SiO}_{2}$ before and after reuse ten times showed the same particle size by transmission electron microscopy (TEM; figure 3).

The reaction of benzaldehyde, aniline and diethylphosphite in the presence of different catalyst was considered as the model reaction. In our present study we screened different catalyst and conditions. Surprisingly, the best was result obtained in using $1.1 \mathrm{~mol} \%$ nano- $\mathrm{SiO}_{2}$ at room temperature under ultrasonication in $15 \mathrm{~min}$. A careful analysis of table 2 reveals the fact that, the reaction afforded 26-96\% yield in the presence 5-20 mol\% of different catalysts. Promising results were obtained with nano- $\mathrm{SiO}_{2}$ in lesser time with better yield and reduced amount of catalyst. The increased catalytic activity of nano- $\mathrm{SiO}_{2}$ over the another catalysts may be attributed to the higher surface area of $\mathrm{SiO}_{2}$.

The catalytic activity of the nano- $\mathrm{SiO}_{2}$ particles was compared with that of the bulk-SiO${ }_{2}$. For this purpose, the reactions were carried out separately under

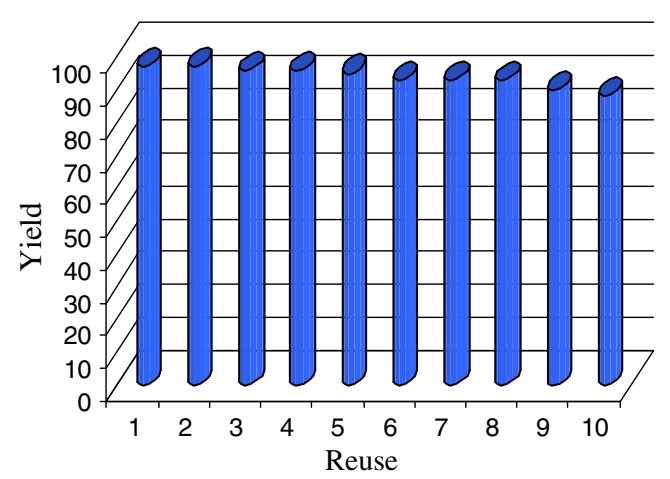

Figure 5. Reuses performance of the catalysts. 
Table 2. Effect of various catalysts and solvent condition on the synthesis of $\alpha$-aminophosphonates.

\begin{tabular}{|c|c|c|c|c|c|c|}
\hline Entry & Catalyst & $\begin{array}{c}\text { Amount of } \\
\text { catalyst }(\mathrm{mol} \%)\end{array}$ & Solvent & $\begin{array}{l}\text { Temp } \\
\left({ }^{\circ} \mathrm{C}\right)\end{array}$ & $\begin{array}{l}\text { Time } \\
(\mathrm{min})\end{array}$ & $\begin{array}{c}\text { Yield }^{\mathrm{a}} \\
(\%)\end{array}$ \\
\hline 1 & $\mathrm{AlCl}_{3}$ & 20 & $\mathrm{EtOH}$ & r. t. & 60 & $30^{15}$ \\
\hline 2 & $\mathrm{ZnCl}_{2}$ & 20 & $\mathrm{EtOH}$ & r. t. & 60 & $26^{15}$ \\
\hline 3 & $\mathrm{YbCl}_{3}$ & 10 & $\mathrm{EtOH}$ & r. t. & 60 & $68^{15}$ \\
\hline 4 & $\mathrm{Yb}(\mathrm{OTf})_{3}$ & 5 & $\mathrm{EtOH}$ & r. t. & 60 & $84^{15}$ \\
\hline 5 & $\mathrm{Yb}(\mathrm{PFO})_{3}$ & 5 & Neat & r. t. & 60 & $92^{16}$ \\
\hline 6 & $\mathrm{MoO} 2 \mathrm{Cl} 2$ & 5 & Neat & 80 & 15 & $87^{17}$ \\
\hline 7 & $\mathrm{Cd}\left(\mathrm{ClO}_{4}\right)_{2} \cdot \mathrm{xH}_{2} \mathrm{O}$ & 5 & Neat & 40 & 45 & $92^{18}$ \\
\hline 8 & ChCl_2ZnCl 2 & 15 & Neat & r. t. & 60 & $96^{19}$ \\
\hline 10 & $\mathrm{FeCl}_{3}^{-}$ & 5 & THF & 60 & 45 & $95^{20}$ \\
\hline 11 & Nano- $\mathrm{SiO}_{2}$ & 1.1 & $\mathrm{H}_{2} \mathrm{O}$ & r.t. & 15 & 97 \\
\hline
\end{tabular}

${ }^{\mathrm{a}}$ Isolated yields

Table 3. Comparative catalytic activity of nano- $\mathrm{SiO}_{2}$ with bulk- $\mathrm{SiO}_{2}$.

\begin{tabular}{lccc}
\hline & \multirow{2}{c}{$\begin{array}{c}\text { Reaction } \\
\text { time (min) }\end{array}$} & ${\mathrm{Nano}-\mathrm{SiO}_{2}}^{\mathrm{Y}}$ & $\mathrm{Bulk}^{\mathrm{a}}-\mathrm{SiO}_{2}$ \\
\cline { 3 - 4 } 1 & 5 & 76 & 33 \\
2 & 10 & 85 & 39 \\
3 & 15 & 97 & 48 \\
4 & 20 & 97 & 52 \\
5 & 25 & 97 & 52 \\
\hline
\end{tabular}

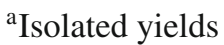

ultrasonication in water with both the catalysts for the appropriate time (table 3 ). The aliquots of the reaction mixture were collected periodically at an interval of $5 \mathrm{~min}$. Table 3 shows the variation of the percentage preparation of $a$-aminophosphonates with time, when nano- $\mathrm{SiO}_{2}(0.0007 \mathrm{~g})$ and bulk- $\mathrm{SiO}_{2}(0.0007 \mathrm{~g})$ were employed as catalysts. It is evident that, the catalytic activity of the nano- $\mathrm{SiO}_{2}$ is much greater than that of the bulk- $\mathrm{SiO}_{2}$. After $15 \mathrm{~min}$, nano- $\mathrm{SiO}_{2}$ showed 97\% preparation of $a$-aminophosphonates as compared to $48 \%$ with bulk- $\mathrm{SiO}_{2}$. The increased catalytic activity of nano- $\mathrm{SiO}_{2}$ over the bulk-SiO${ }_{2}$ may be attributed to the higher surface area of $\mathrm{SiO}_{2}$.

Table 4. Synthesis of $a$-aminophosphonates derivatives catalysed by nano- $\mathrm{SiO}_{2}$.

\begin{tabular}{lllccccc}
\hline Entry & \multicolumn{1}{c}{ Aldehyde } & \multicolumn{1}{c}{ Amine } & Product & Time $(\mathrm{min})$ & Yield $(\%)^{\mathrm{a}, \mathrm{b}}$ & Obs. mp $\left({ }^{\circ} \mathrm{C}\right)$ & Lit. mp $\left({ }^{\circ} \mathrm{C}\right)$ \\
\hline 1 & $\mathrm{C}_{6} \mathrm{H}_{5} \mathrm{CHO}$ & $\mathrm{C}_{6} \mathrm{H}_{5} \mathrm{NH}_{2}$ & $\mathbf{4 a}$ & 15 & 97 & $88-90$ & $91-93^{21}$ \\
2 & $\mathrm{C}_{6} \mathrm{H}_{5} \mathrm{CHO}$ & $2-\mathrm{ClC}_{6} \mathrm{H}_{4} \mathrm{NH}_{2}$ & $\mathbf{4 b}$ & 15 & 95 & $84-86$ & $85-86^{22}$ \\
3 & $\mathrm{C}_{6} \mathrm{H}_{5} \mathrm{CHO}$ & $3-\mathrm{ClC}_{6} \mathrm{H}_{4} \mathrm{NH}_{2}$ & $\mathbf{4 c}$ & 10 & 93 & $119-121$ & $122-123^{23}$ \\
4 & $\mathrm{C}_{6} \mathrm{H}_{5} \mathrm{CHO}$ & $4-\mathrm{ClC}_{6} \mathrm{H}_{4} \mathrm{NH}_{2}$ & $\mathbf{4 d}$ & 15 & 96 & $114-116$ & $111-113^{24}$ \\
5 & $\mathrm{C}_{6} \mathrm{H}_{5} \mathrm{CHO}$ & $4-\mathrm{FC}_{6} \mathrm{H}_{4} \mathrm{NH}_{2}$ & $\mathbf{4}$ & 20 & 94 & $109-111$ & $111-112^{26}$ \\
6 & $\mathrm{C}_{6} \mathrm{H}_{5} \mathrm{CHO}$ & $4-\mathrm{NO}_{2} \mathrm{C}_{6} \mathrm{H}_{4} \mathrm{NH}_{2}$ & $\mathbf{4 f}$ & 15 & 97 & $148-150$ & $145-146^{22}$ \\
7 & $\mathrm{C}_{6} \mathrm{H}_{5} \mathrm{CHO}$ & $4-\mathrm{CH}_{3} \mathrm{C}_{6} \mathrm{H}_{4} \mathrm{NH}_{2}$ & $\mathbf{4 g}$ & 15 & 93 & $115-116$ & $117-118^{22}$ \\
8 & $\mathrm{C}_{6} \mathrm{H}_{5} \mathrm{CHO}$ & $4-\mathrm{OCH}_{3} \mathrm{C}_{6} \mathrm{H}_{4} \mathrm{NH}_{2}$ & $\mathbf{4 h}$ & 20 & 92 & $80-82$ & $78-80^{25}$ \\
9 & $4-\mathrm{OCH}_{3} \mathrm{C}_{6} \mathrm{H}_{4} \mathrm{CHO}$ & $4-\mathrm{NO}_{2} \mathrm{C}_{6} \mathrm{H}_{4} \mathrm{NH}_{2}$ & $\mathbf{4 i}$ & 20 & 94 & $103-105$ & $107-108^{26}$ \\
10 & $2-\mathrm{NO}_{2} \mathrm{C}_{6} \mathrm{H}_{4} \mathrm{CHO}$ & $\mathrm{C}_{6} \mathrm{H}_{5} \mathrm{NH}_{2}$ & $\mathbf{4 j}$ & 20 & 92 & $158-159$ & $155-156^{27}$ \\
11 & $4-\mathrm{NO}_{2} \mathrm{C}_{6} \mathrm{H}_{4} \mathrm{CHO}$ & $\mathrm{C}_{6} \mathrm{H}_{5} \mathrm{NH}_{2}$ & $\mathbf{4 k}$ & 15 & 91 & $122-124$ & $125-126^{21}$ \\
12 & $4-\mathrm{ClC}_{6} \mathrm{H}_{4} \mathrm{CHO}$ & $\mathrm{C}_{6} \mathrm{H}_{5} \mathrm{NH}_{2}$ & $\mathbf{4 l}$ & 10 & 95 & $77-78$ & $75-76^{28}$ \\
13 & $4-\mathrm{CH}_{3} \mathrm{C}_{6} \mathrm{H}_{4} \mathrm{CHO}$ & $\mathrm{C}_{6} \mathrm{H}_{5} \mathrm{NH}_{2}$ & $\mathbf{4 m}$ & 20 & 93 & $64-66$ & $66-67^{21}$ \\
14 & $4-\mathrm{OCH}_{3} \mathrm{C}_{6} \mathrm{H}_{4} \mathrm{CHO}$ & $\mathrm{C}_{6} \mathrm{H}_{5} \mathrm{NH}_{2}$ & $\mathbf{4 n}$ & 10 & 96 & $104-106$ & $101-102^{25}$ \\
\hline
\end{tabular}

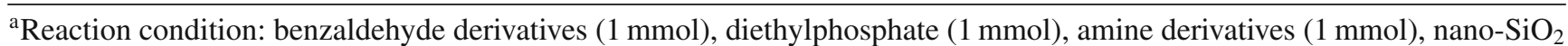
$(0.0007 \mathrm{~g})$ at room temperature in water under ultrasonication

${ }^{\mathrm{b}}$ Yield refers to isolated product 


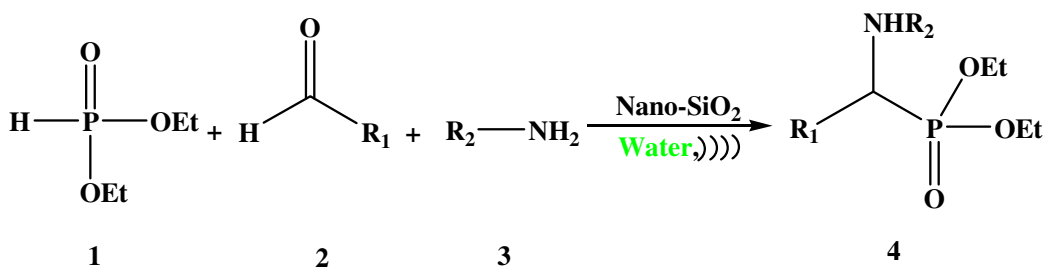

Scheme 2. Synthesis of derivatives 4 in the presence of nano- $\mathrm{SiO}_{2}$.

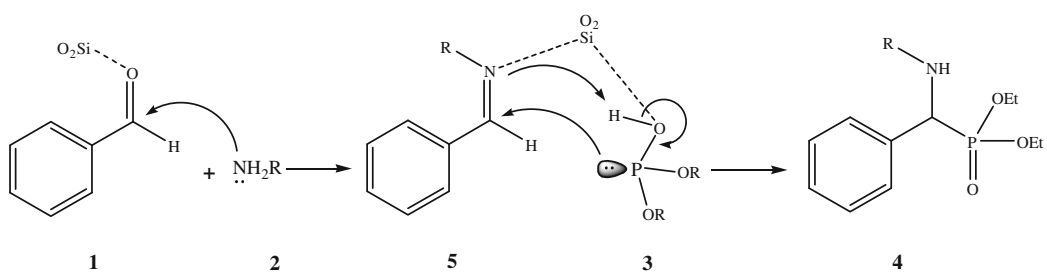

Scheme 3. Suggested mechanism for the preparation of $a$-aminophosphonates.

After optimization of the reaction conditions, to delineate this approach, particularly in regard to library construction, this methodology was evaluated by using different amines, variety of different substituted benzaldehydes and of diethylphosphate in the presence of nano- $\mathrm{SiO}_{2}$ under similar conditions. As can be seen from table 4, electronic effects and the nature of substituents on the aromatic ring did not show obvious effects in terms of yields under the reaction conditions. The three-component reaction proceeded smoothly and was completed in 10-20 min. Benzaldehyde and other aromatic aldehydes containing electron-withdrawing groups or electron-donating were employed and reacted well to give the desired products in excellent yields with high purity (scheme 2).

The proposed mechanism for the role of nano$\mathrm{SiO}_{2}$ as a catalyst is shown in scheme 2. In this mechanism the formation of $a$-aminophosphonates 4 from the three-component reaction of benzaldehyde, amine, and dialkyl phosphite was proposed to involve two steps: Knoevenagel condensation of the benzaldehyde with amine takes place with elimination of the hydroxide anion and formation of product 5 followed by phospha-Michael addition of dialkyl phosphate $\mathbf{3}$ to electron deficient Knoevenagel adduct 5 leading to the corresponding $a$-aminophosphonates product $\mathbf{4}$ (scheme 3).

\section{Conclusions}

In conclusion, the obtained results describe a novel, and simple method for the synthesis of $a$ aminophosphonates via one-pot, three-component reaction using nano- $\mathrm{SiO}_{2}$ as catalyst at roomtemperature in water under ultrasonication with high product yields. This nanoparticles catalyst provides a new way for continuous processes, because of its simple recyclability. From a scientific point, our results expand the application of 'free' nanoparticles. This catalyst would be helpful to understand the advantageous combination of the properties of heterogeneous catalysis and the development of new catalytic systems.

\section{References}

1. Anastas $\mathrm{P} \mathrm{T}$ and Williamson $\mathrm{T} 1998$ Green chemistry: Frontier in benign chemical synthesis and process (Oxford, UK: Oxford University Press)

2. Polshettiwar V, Luque R, Fihri A, Zhu H, Bouhrara M and Basset J 2011 Chem. Rev. 1113036

3. Herrerias C I, Yao X, Li Z and Li C 2007 Chem. Rev. 1072546

4. Kljin J E and Engberts J B 2005 N. Nature $\mathbf{4 3 5} 746$

5. Pacchioni G 2000 Surf. Rev. Lett. 7277

6. Kalor A, Cox D and Zakin M R 1988 Adv. Chem. Phys. 70211

7. Hosseini-Sarvari M 2011 Catal. Lett. 141347

8. Koukabi N, Kolvari E, Khazaei A, Zolfigol M A, Shirmardi-Shaghasemi B and Khavasi H R 2011 Chem. Commun. 479230

9. Allen M C, Fuhrer W, Tuck B, Wade R and Wood J M 1989 J. Med. Chem. 321652

10. Nakamura S, Hayashi M, Hiramatsu Y, Shibata N, Funahashi Y and Toru T 2009 J. Am. Chem. Soc. 131 18240

11. Sharghi H, Nasseri M A and Hassani Nejad A 2003 J. Mol. Catal. A: Chem. 20653

12. Sharghi H, Hassani Nejad A R and Nasseri M A 2004 New J. Chem. 28946 
13. Nasseri M A, Kazemi F and Sharghi H 2004 Synthesis 2205

14. (a) Yu M, Lin J and Fang J 2005 Chem. Mater. 17 1783; (b) Musi S, Filipovi-Vincekovi N and Sekovani L 2011 Brazil. J. Chem. Eng. 28 89; (c) Wei-Jie Y, Zhen-Feng Z and Chun-Lei G 2008 Key Eng. Mater. 368787

15. Tang J, Wang L, Wang W, Zhang L, Wu S and Mao D 2011 J. Fluorine Chem. 132102

16. Noronha R G, Romão C C and Fernandes A C 2011 Catal. Commun. 12337

17. Dindulkar S D, Reddy M V and Jeong Y T 2012 Catal. Commun. 17114

18. Disale S T, Kale S R, Kahandal S S, Srinivasan T G and Jayaram R V 2012 Tetrahedron Lett. 532277

19. Sheykhan M, Mohammadnejad H, Akbari J and Heydari A 2012 Tetrahedron Lett. 532959
20. Rezaei Z, Firouzabadi H, Iranpoor N, Ghaderi A, Jafari M R, Jafari A A and Zare H R 2009 Eur. J. Med. Chem. 444266

21. Qian C T and Huang T 1998 J. Org. Chem. 634125

22. Firouzabadi H, Iranpoor N and Sobhani S 2004 Synthesis 2692

23. Bhanushali M J, Nandurkar N S, Jagtap S R and Bhanage B M 2009 Synth. Commun. 39845

24. Sobhani S and Vafaee A 2009 Synthesis 1909

25. Wu J, Sun W, Xia H G and Sun X Y 2006 Org. Biomol. Chem. 91663

26. Srikant B and Asit K C 2007 J. Org. Chem. 721263

27. Vinu A, Kalita P, Balasubramanian V V, Oveisi H, Selvan T, Mano A, Chari M A and Subba Reddy B V 2009 Tetrahedron Lett. 507132

28. Manabe K and Kobayashi S 2000 Chem. Commun. 669 